\title{
Study of ossification in long bones of aborted human fetuses of various weeks of gestation by Alcian blue stain
}

\author{
Arpan Haldar ${ }^{1}$, Manisha R. Gaikwad ${ }^{2 *}$, Amit P. Tirpude ${ }^{3}$, Provas Banerjee ${ }^{4}$, Soumya C Bhattacharya ${ }^{5}$ \\ ${ }^{1}$ Senior Resident, ${ }^{\mathbf{2}}$ Additional Professor \& HOD, ${ }^{\mathbf{3}}$ Asssitant Professor, ${ }^{\mathbf{1 , 2 , 3}}$ All India Institute of Medical Sciences, Bhubaneswar, \\ Odisha, ${ }^{4}$ Professor \& HOD, Dept. of Anatomy, Hi-tech Medical College, Rourkela, Odisha, ${ }^{5}$ Professor \& HOD, Dept. of \\ Anatomy, ESI Post Graduate Institute of Medical Sciences and Research, Joka, Kolkata, India \\ *Corresponding Author: \\ Email: manishagaikwad6719@yahoo.in
}

Received: $15^{\text {th }}$ February, 2018

Accepted: $08^{\text {th }}$ March, 2018

\begin{abstract}
Bone consists of bone cells and interstitial matrix, which is further divided into organic and inorganic. Four types of bone cells are present- osteoprogenitor cells, osteoblasts, osteocytes \& osteoclasts. The bones are arranged in Haversian system or secondary osteons, which consists of central Haversian canal surrounded by concentric lamellae of bony tissue. These Haversian canals or the canals of Havers are in direct communication with medullary cavity also communicate with the surface of bone via Volkmann's canal. Bone ossification in human foetuses is not widely studied. The information on primates is considered for comparison. In the present study attempts have been made to study the long bone ossification pattern in aborted human foetus between gestational ages 10 weeks to 32 weeks of gestation by Alcian Blue stain. The stages of cartilage transformation to bones are seen in six stages. The present study was aimed to evidence the human foetus long bones ossification at various weeks of gestation. The emanated information may enrich the existing global information on human foetuses ossification. From each foetus of different gestational weeks longitudinal and transverse sections of 7 long bones (clavicle, humerus, radius, ulna, femur, tibia, and fibula) were taken into study. All of them were stained by Alcian Blue stain.
\end{abstract}

Keywords: Osteoprogenitor cells, Osteoblasts, Alcian Blue, Haversian canal, Volkmann's canal.

\section{Introduction}

The bone consists of outer compact and inner spongy part (cancellous bone), composed of a network of trabeculae. Each bone is covered externally by periosteum and internally by endosteum, which lines a medullary cavity filled with bone marrow. The periosteum consists of outer fibrous layer composed of collagen fibres, inner cellular and vascular layer known as osteogenic layers containing osteoblasts, which deposit layers of bones on the outer surface of the young bone. Bone consists of bone cells and interstitial matrix, which is further divided into organic and inorganic. ${ }^{1}$ Four types of bone cells are osteoprogenitor cells, osteoblasts, osteocytes \& osteoclasts. The interstitial matrix mainly consists of the bone marrow cavity in the spongy bone. Osteoprogenitor cells are derived from mesenchyme and are committed to form bone tissue. They lie beneath the periosteum and endosteum which are inducible in various connective tissues (heterotropic bone formation). Committed osteoprogenitor cells proliferate by mitosis and differentiate into osteoblasts in more vascularised area with sufficient oxygen concentration or into chondroblasts when oxygen tension is low. Osteoblasts are developed from the osteoprogenitor cells. The osteocytes are derived from the osteoblasts. The osteoclasts are derived from the fusion of mononuclear phagocyte system (MPS) of bone marrow. ${ }^{2}$ The bones are arranged in Haversian system or secondary osteons, which consists of central Haversian canal surrounded by concentric lamellae of bony tissue. These Haversian canals or the canals of Havers are in direct communication with medullary cavity which also communicates with the surface of bone via Volkmann's canal. Bone grows in length by interstitial method and in width by appositional method. The transition between epiphyseal cartilage and new bone is seen as epiphyseal plate. ${ }^{3}$ Bone ossification in human fetuses is not widely studied. The information on primates is considered for comparison. ${ }^{4}$ In the present study attempts have been made to study the long bone ossification pattern in aborted human fetuses between gestational ages 10 weeks to 32 weeks. Mother's nutritional status, use of steroids in pregnancy \& any endocrinopathies like hypopituitarism or hypothyroidism affect the bone ossification and mineralization..$^{5}$ This could be better projected in the Odisha population which is geographically and socio-economically different from the other Indian states. Osteogenesis of foetuses, expressed on the hormonal level affecting mineral metabolism and placental activity of mother have a complex relationship. Our study by Alcian Blue stain will help to differentiate between the Alizarin Red-S stain and Haematoxylin and Eosin (H\&E) stain previously used in small vertebrates. ${ }^{6}$ Ultimately this study may provide future guidelines in forensic medicine to determine feticide, toxicity studies, teratogenic effect of drugs affecting bones mineralization, study of 
xenobiotics and will give scientific evidence for future studies like foetal bone grafting. ${ }^{7}$

\section{Materials and Methods}

The study was an observational and cross sectional conducted at Hi- Tech Medical College \& Hospital, Bhubaneswar, Odisha, India by the Department of Anatomy in collaboration with Department of Obstetrics \& Gynaecology. It covered a time span from November 2011 to June 2013 on twenty four aborted human foetuses without obvious congenital anomaly. The gestational age of fetuses ranged between 10 weeks to 32 weeks collected within 6 hours of delivery by therapeutic legal abortions, intrauterine deaths and stillbirths. Fetuses were arbitrarily divided into groups of biweekly gestational age by duration of amenorrhoea from medical records \& ultrasound fetometry after receipt of informed consent from mother and legal guardians. The foetal age was measured by Crown-Rump Length (CRL) in foetuses of 10-20 weeks gestational age whereas Crown-Heel Length (CHL) was more accurate in foetuses of 20-32 weeks. Consents were taken by principal researcher himself. All participants were clearly explained that they reserve the right to refuse to participate in the study. A copy of the consent form was given to each legal guardians/parents. The study was continuously being monitored by the Institutional ethics committee during the study period. The rules and guidelines for disposal of human anatomical waste were strictly followed during the study. Two foetuses were collected from each week, fixed in 10\% Formalin and then processed for histology of long bone by Alcian Blue stain. Bones were dissected under dissecting microscope. Then they were decalcified by $3 \%$ Hydrochloric acid $(\mathrm{HCl})$ solution in $70 \%$ alcohol for a few days, and 20 drops of concentrated $\mathrm{HCl}$ was added daily for about 5-10 days according to the amount of calcium present. Decalcification was tested by pricking a needle in epiphyseal end of bone and also by adding ammonia solution which caused the solution to turn cloudy. After decalcification the tissue was placed in neutral $70 \%$ alcohol, which was changed daily. Neutralisation was then tested by blue litmus paper test. Few drops of saturated aqueous solution of lithium carbonate were added to the alcohol to accelerate the process of neutralisation. After decalcification, the specimens were washed in running tap water to avoid formation of precipitates. The decalcified bones were cut in $5 \mathrm{~mm}$ thickness. Both transverse and longitudinal sections for each bone of foetuses of individual periods of gestation were fixed in $10 \%$ formalin. A few drops of glycerol were added as formalin itself produces little shrinkage. After fixation by formalin, the tissues were transferred to $30 \%, 50 \%, 70 \%, 90 \%$ and Absolute alcohol each for 30 minutes. Then the tissues were put in xylol for 24 hours to clear the residual alcohol. The decalcified bones were processed for paraffin sections by tissue blocking (Paraffin Embedding). 3 pots of hard paraffin were taken; paraffin was melted in the incubator at 56 degrees, as hard paraffin is ideal for materials which are to be cut in thin sections about $5 \mu$. The tissue was put in the first pot containing equal parts of paraffin and xylol and then changed to second and third pots containing only fresh melted paraffin at 90 minutes interval. Then the tissues were mounted in fresh melted paraffin with L-Block. The L-Block was then trimmed to a rectangular shape. Then the L-Block was fixed with the block holder (choke) and the block holder was clamped in the rotary microtome. $5 \mu$ sections were cut in rotary microtome. The microtome was revolved at $40 \mathrm{rpm}$ and ribbon was formed. Then the ribbon was put in tissue flotation bath at 56 degree Celsius. Albuminised slide was prepared by putting a drop of Mayor's albumin (equal parts of glycerine and egg white) and spreading it uniformly by rubbing with finger. The piece of ribbon was then taken on the slide and dried at room temperature. The slide was then put in the warming table. When the paraffin melted the slide was put into xylol for 2-3 minutes because xylol removes paraffin. Then the tissue was put in decreasing grades of alcohol (Absolute alcohol, 90\%, 70\%, 50\% and 30\%) then was washed with distilled water. These slides were dipped in Alcian blue solution for 30 minutes. Slides were washed in running tap water for 2 minutes and then rinsed in distilled water. The counterstaining was done by Nuclear Fast Green solution for 5 minutes and then washed in running tap water for 1 minute. 2-3 drops of $1 \%$ acid alcohol (1cc $\mathrm{HCl}$ in $75 \%$ alcohol) was added to remove the excess stain beyond the nucleus. Then the slide was washed with absolute alcohol for a few seconds and lastly the slide was placed in xylol. The slide was then taken out from xylol and then put in 1-2 drops of DPX (Adhesive agent) and a cover slip was put on it and pressed slightly so that air bubbles were removed ${ }^{(8)}$. Sections were then seen in light microscope under low power 10X followed by high power 40X magnification. Thereafter photomicrographs were taken by camera using microscope adapter.

Preparation of Alcian Blue Stain: 3\% acetic acidsolution was made by $3 \mathrm{ml}$ glacial acetic acid in 97 $\mathrm{ml}$ distilled water. Alcian Blue solution was then made by adding $1 \mathrm{gm}$ Alcian blue in $100 \mathrm{ml}$ of $3 \%$ acetic acid. The solution was mixed well. The $\mathrm{pH}$ was adjusted to 2.5 using acetic acid by litmus paper.

Then Counterstain i.e. nuclear fast green was prepared by slightly boiling $0.1 \mathrm{gm}$ of nuclear fast green with $5 \mathrm{gm}$ of aluminium sulphate in $100 \mathrm{ml}$ distilled water. It was then cooled and filtered. ${ }^{8}$

\section{Results}

In the longitudinal sections, the stages of cartilage transformation to bones is seen. Endochondral 
ossification of epiphyseal growth plate was seen in six stages:

1. Zone of Resting Cartilage: Typical hyaline cartilage with chondrocytes were arranged in small clusters surrounded by a large amount of moderately stained matrix.

2. Zone of Proliferation: The clusters of cartilagecells were seen to undergo successive mitotic divisions to form columns of chondrocytes separated by strongly stained matrix.

3. Zone of Maturation: Cell division was not seen and chondrocytes were increased in size.

4. Zone of Hypertrophy and Calcification: The chondrocytes were seen to be greatly enlarged and vacuolated, the matrix became calcified.

5. Zone of Cartilage Degeneration: The chondrocytes were seen to degenerate and the lacunae of the calcified matrix were invaded by osteogenic cells and capillaries from the marrow cavity of the diaphysis.

6. Zone of Ossification: The osteogenic cells were seen to differentiate into osteoblasts which congregated on the surface of the spicules of calcified cartilage matrix where they commence bone formation. ${ }^{9}$

In the longitudinal sections (LS) the cartilage cells with prominent nuclei were seen, then gradually due to decreased vascularity the chondrocytes died and bone formation started. The osteoblasts gradually converted to osteocytes in older ages of gestation which were seen in the lacunae. The periosteum was seen to underlying bone by bundles of collagen fibres called the Sharpey's fibres which penetrated the whole thickness of cortical bone. The osteoclasts were seen to be present in Howship's lacunae which helped in bone resorption. The bony spicules and plates of calcified cartilage matrix were seen with the blood sinusoids. The haemopoietic tissue was seen in the marrow cavity which then formed the arterioles. ${ }^{10}$

In the transverse sections (TS) the Primitive Haversian Canals gets gradually converted into Haversian system containing central canal and vessels. The bones were arranged in Haversian system or secondary osteons, which consists of central Haversian canal surrounded by concentric lamellae of bony tissue. The Haversian canals or canals of Havers were in direct communication with medullary cavity and were in communication with the surface of bone via Volkmann' canals or primary osteons. The Cement line was seen between the two osteons.

Compact Bone \& Cartilage were stained Deep Blue, Bone Marrow Cavity was stained Light Blue and Nuclei was stained Black. ${ }^{11}$

\section{Discussion}

Mother's nutritional status, use of steroids in pregnancy $\&$ any endocrinopathies like hypopituitarism or hypothyroidism which may affect the bone ossification and mineralization could be better projected in the Odisha population geographically and socioeconomically different from the other Indian states. ${ }^{12}$ Previous works on the use of common H/E stain in small vertebrates could be correlated well and may help to differentiate the $\mathrm{H} / \mathrm{E}$ stain from other special stain like Masson's Trichome, Von Kossa, PAS, Mallory Azan, Van Geison etc. used for long bones. ${ }^{13}$ This study needs further enrichment by scanning electron microscope, immune-histochemistry studies as well as by radiographic and ultrasound methods. ${ }^{14}$ This study may provide enough information relating to human fetal long bone ossification to determine foeticide..$^{15}$ At large it will act as future guidelines in forensic medicine, toxicity studies and teratogenic effect of drugs affecting bones mineralization and study of xenobiotics. ${ }^{16}$ Ultimately this study may provide scientific evidence for future studies including foetal bone grafting. ${ }^{17}$

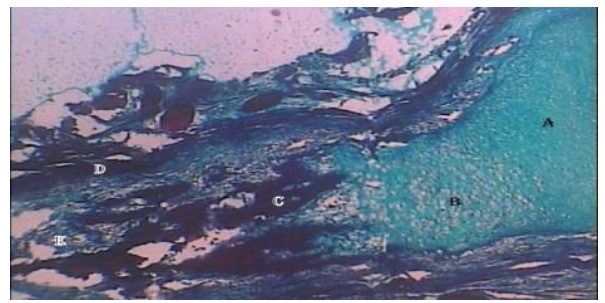

Fig. 1: Alcian Blue stain of LS Section of 12 weeks Fibula showing A-Zone of Proliferation, B-Zone of Hypertrophy \& Calcification, C-Zone of Ossification, D-Marrow Cavity

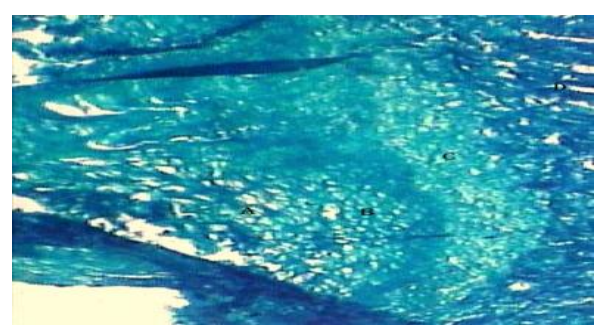

Fig. 2: Alcian Blue stain of LS Section of 24 weeks Radius showing A-Zone of Proliferation, B-Zone of Hypertrophy \& Calcification, C-Zone of Maturation, D-Zone of Ossification

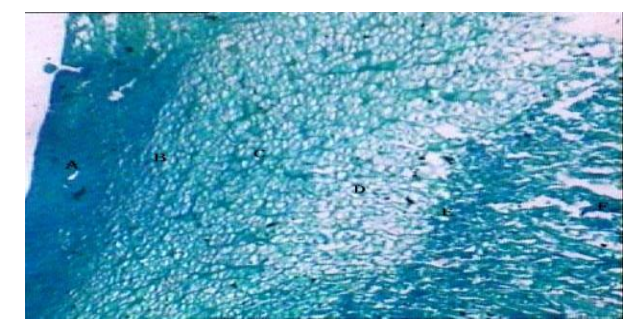

Fig. 3: Alcian Blue stain of LS Section of 14 weeks Fibula showing A-Zone of Reserve Cartilage, B-Zone of Proliferation, C-Hypertrophy \& Calcification, DZone of Cartilage Degeneration, E-Zone of Maturation, F-Zone of Ossification 


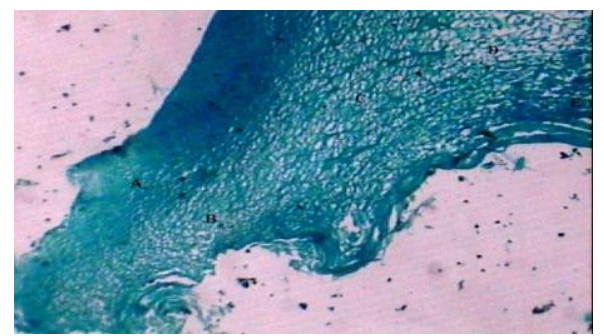

Fig. 4: Alcian Blue stain of LS Section of 12 weeks Radius showing A-Zone of Reserve Cartilage, B-Zone of Proliferation, C-Zone of Hypertrophy \& Calcification, D-Zone of Cartilage Degeneration, EZone of Ossification

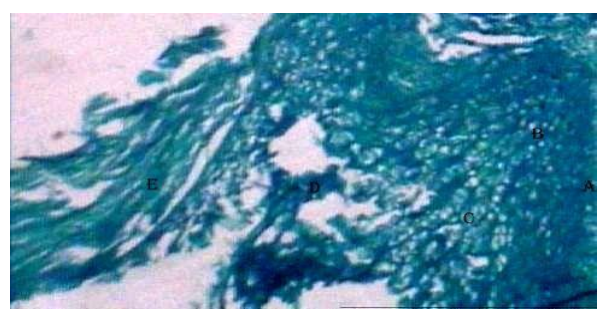

Fig. 5: Alcian Blue stain of LS Section of 10 weeks Femur showing A-Zone of Reserve Cartilage, B-Zone of Proliferation, C-Zone of Hypertrophy \& Calcification, D-Zone of Cartilage Degeneration, EZone of Ossification)

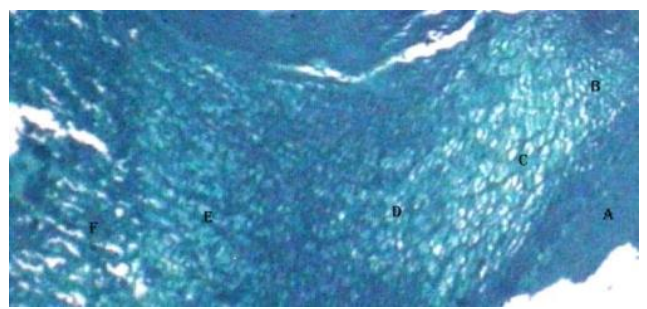

Fig. 6: Alcian Blue stain of LS Section of 28 weeks Fibula showing A-Zone of Reserve Cartilage, B-Zone of Proliferation, C-Zone of Maturation, D-Zone of Hypertrophy \& Calcification, E-Zone of Cartilage Degeneration, F-Zone of Ossification

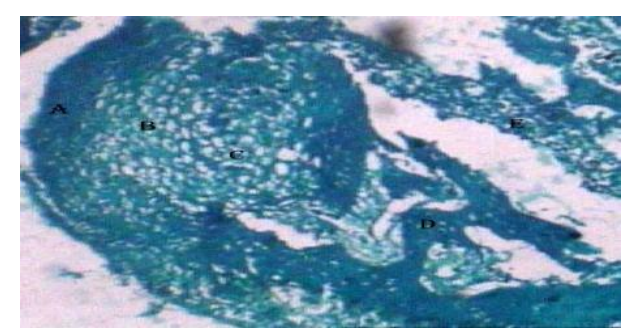

Fig. 7: Alcian Blue stain of LS Section of 12 weeks Ulna showing A-Zone of Reserve Cartilage, B-Zone of Proliferation, C-Zone of Hypertrophy \& Calcification, D-Zone of Ossification, E-Bone Marrow Cavity

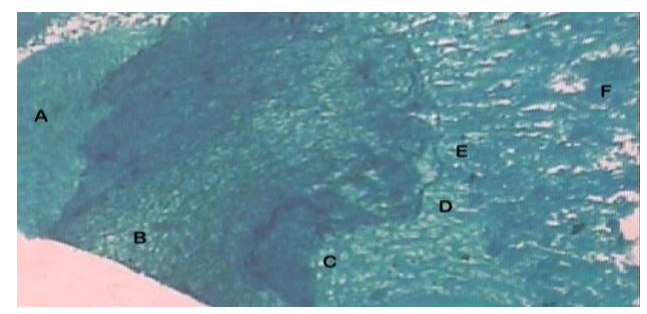

Fig. 8: Alcian Blue stain of LS Section of 16 weeks Tibia showing A-Zone of Reserve Cartilage, B-Zone of Proliferation, C-Zone of Maturation, D-Zone of Hypertrophy \& Calcification, E-Zone of Cartilage Degeneration, F-Zone of Ossification

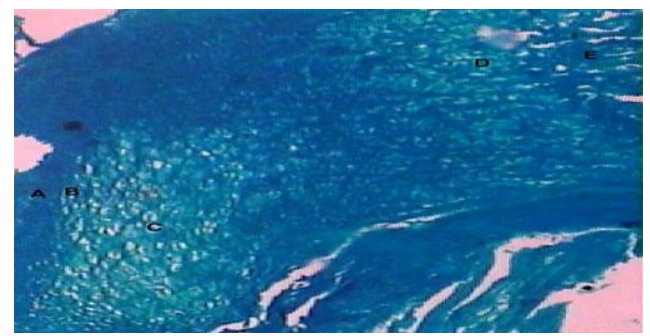

Fig. 9: TS of Alcian Blue stain of LS Section of 24 weeks Radius showing A-Zone of Reserve Cartilage, B-Zone of Proliferation, C-Zone of Maturation, DZone of Hypertrophy \& Calcification, E-Zone of Ossification

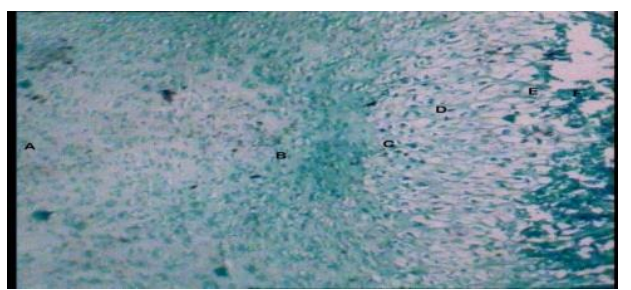

TS of Alcian Blue stain of LS Section of 22 weeks Radius showing A-Zone of Reserve Cartilage, B-Zone of Proliferation, C-Zone of Maturation, D-Zone of Hypertrophy \& Calcification, E-Zone of Cartilage Degeneration, F-Zone of Ossification

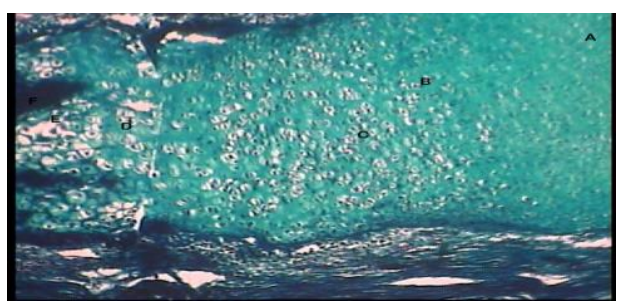

AlcianBlue stain of LS Section of 14 weeks Fibula showing A-Zone of Reserve Cartilage, B-Zone of Proliferation, C-Zone of Maturation, D-Zone of Hypertrophy \& Calcification, E-Zone of Cartilage Degeneration, F-Zone of Ossification 


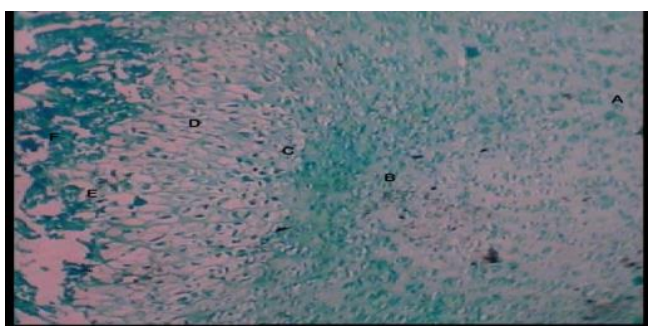

AlcianBlue stain of LS Section of 22 weeks Fibula showing A-Zone of Reserve Cartilage, B-Zone of Proliferation, C-Zone of Maturation, D-Zone of Hypertrophy \& Calcification, E-Zone of Cartilage Degeneration, F-Zone of Ossification

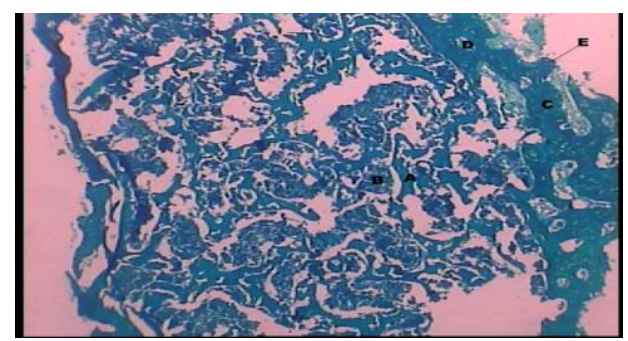

Alcian Blue stain of TS Section of 12 weeks Femur showing A-Bony Spicules,

B-Bone Marrow Cavity, C-Compact Bone, DVolkmann Canals, E-Perichondrium

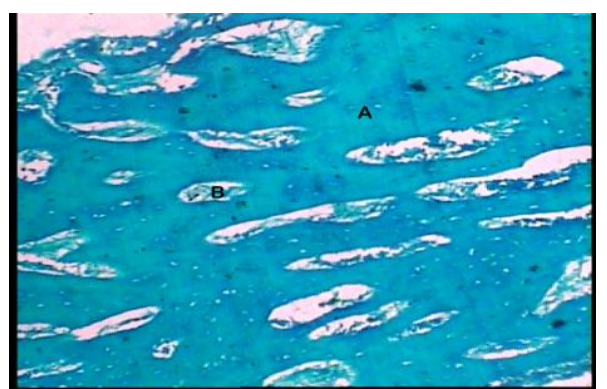

Alcian Blue stain of LS Section of 20 weeks Clavicle showing A-Compact Bone, B-Haversian System Formation

\section{Conclusion}

The present study was aimed to study the human foetus long bones ossification at various weeks of gestation. The emanated information may enrich the existing global information on human foetuses ossification. Our study of ossification centers in long bones will substantiate the findings of the connective tissue, skeletal disorder. Primate models of foetal allogenic bone transplantation and intrauterine repair of skeletal anomalies in man may provide future guidelines.

\section{Conflicts of Interests: None}

\section{References}

1. Carlson BM (2009). Human Embryology and Developmental Biology (4th Ed.) Philadelphia: Saunders Elsevier.

2. Hamilton JW, Boyd JD, Mossman HW (1972). Human Embryology: Prenatal Development of Form and Function (4th Ed.) Cambridge: W Heffer and Sons.

3. Moore KL, Persaud TVN, Torchia MG (2011). The Developing Human: Clinically Oriented Embryology (9th Ed.) Philadelphia: Saunders Elsevier.

4. Dingerkus G, Uhler LD (1977). Enzyme clearing of alcian blue stained whole small vertebrates for demonstration of cartilage. Stain Technol. Jul;52 (4):22932.

5. Pryse-Davies J, Smitham JH, Napier KA (1974). Factors influencing development of secondary ossification centres in the fetus and newborn. A post-mortem radiological study. Arch Dis Child. Jun; 49(6):425-31

6. Miller DM, Tarpley J (1996). An automated double staining procedure for bone and cartilage. Biotech Histochem. Mar; 71(2):79-83.

7. Kiernan J (2008). Histological and Histochemical Methods Theory and Practice (4th Ed.) Oxfordshire: Scion Publishing.

8. Ullah M (1980). Histology \& Genetics: Theoretical \&Applied. Meerut: Kedar Nath Ram Nath.

9. Mescher AL. Junqueira's (2009). Basic Histology: Text \&Atlas (12th ed.) New York: McGraw-Hill Education.

10. Ross MH, Pawlina W. (2011). Histology A Text and Atlas (6th ed.) Philadelphia: Lippincott Williams \& Wilkins.

11. Standring S (2008). Gray's Anatomy: The Anatomical Basis of Clinical Practice. (40th ed.) Edinburgh: Churchill Livingtone.

12. Alphonse R. Burdi (1965). Toluidine Blue-Alizarin Red S Staining of Cartilage and Bone in Whole-Mount Skeletons in Vitro Biotechnic \& Histochemistry, Vol. 40, No. 2:Pages 45-8.

13. Oshima A (1971). Ossification of the hearing apparatus in the Japanese fetus studied by ultra-soft $\mathrm{x}$-rays and alizarin red bone staining. Nihon Jibiinkoka Gakkai Kaiho. Aug;74(8):1226-34.

14. Gentili P, Giorlandino C, Parapella P (1982). Echo graphic evaluation of principal ossification centres in the foetuses. Minerva Ginecol. Sept;34(9):701-4.

15. Kjar I (1974). Skeletal maturation of the human fetus assessed radiographically on the basis of ossification sequences in the hand and foot Am J Phys Anthropol. Mar;40(2):257-75.

16. Menegola E, Broccia ML, Di Renzo F, Giavini E. (2002) Comparative study of sodium valproate-induced skeletal malformations using single or double staining methods. Reprod Toxicol. Nov-Dec;16(6):815-23.

17. Michejda M, Bacher J, Kuwabara T, Hodgen GD (1981). In utero allogeneic bone transplantation in primates: roentgenographic and histological observations. J of Transplantation Aug; 32(2):96-100.

\section{Acknowledgement}

The corresponding author acknowledges the support and guidance received from Prof. Dr. Dipti Basu. 